\title{
Electrospray Printing of Polyimide Films Using Passive Material Focusing
}

Bryce J. Kingsley, Emma E. Pawliczak, Thomas R. Hurley, Paul R. Chiarot

Department of Mechanical Engineering

State University of New York at Binghamton

Binghamton, NY 13902, USA

*pchiarot@binghamton.edu

\section{SUPPLEMENTARY INFORMATION}

Table S1: Mass concentration and flow rate compositions.

\begin{tabular}{|c|c|c|}
\hline Mass Delivery Rate $[\mu \mathrm{g} / \mathrm{min}]$ & Mass Loading [wt. \%] & Flow Rate $[\boldsymbol{\mu L} / \mathbf{m i n}]$ \\
\hline 24 & 1 & 2.5 \\
\hline 36 & 3 & 1.23 \\
\hline 48 & 2 & 2.5 \\
\hline 73 & 3 & 2.5 \\
\hline 87 & 4 & 2.2 \\
\hline 99 & 4 & 2.5 \\
\hline 109 & 4 & 2.75 \\
\hline 118 & 4 & 3 \\
\hline
\end{tabular}

Table S2: Film breakdown testing raw data.

\begin{tabular}{|c|c|c|}
\hline Film Thickness $[\boldsymbol{\mu m}]$ & Breakdown Potential [V] & Breakdown Field Strength [V/ $\boldsymbol{\mu m}]$ \\
\hline 4.1 & 400 & 99 \\
\hline 4.1 & 495 & 122 \\
\hline 4.1 & 370 & 91 \\
\hline 4.1 & 495 & 122 \\
\hline 4.1 & 420 & 104 \\
\hline 6.0 & 500 & 83 \\
\hline 6.0 & 640 & 107 \\
\hline 6.0 & 650 & 108 \\
\hline 6.0 & 850 & 142 \\
\hline 6.0 & 800 & 133 \\
\hline 7.1 & 820 & 115 \\
\hline 7.1 & 1010 & 142 \\
\hline 7.1 & 575 & 81 \\
\hline 7.1 & 815 & 114 \\
\hline 7.1 & 900 & 126 \\
\hline 4.1 & 410 & 101 \\
\hline
\end{tabular}




\begin{tabular}{|c|c|c|}
\hline 4.1 & 475 & 117 \\
\hline 4.1 & 430 & 106 \\
\hline 4.1 & 500 & 123 \\
\hline 4.1 & 400 & 99 \\
\hline 6.0 & 820 & 137 \\
\hline 6.0 & 620 & 103 \\
\hline 6.0 & 760 & 127 \\
\hline 6.0 & 540 & 90 \\
\hline 6.0 & 510 & 85 \\
\hline 7.1 & 770 & 108 \\
\hline 7.1 & 620 & 87 \\
\hline 7.1 & 590 & 83 \\
\hline $\begin{array}{l}7.1 \\
\end{array}$ & 515 & 72 \\
\hline 7.1 & 660 & 93 \\
\hline 7.5 & 150 & 20 \\
\hline 7.5 & 175 & 23 \\
\hline 7.5 & 225 & 30 \\
\hline 7.5 & 150 & 20 \\
\hline 7.5 & 270 & 36 \\
\hline 13.0 & 200 & 15 \\
\hline 13.0 & 350 & 27 \\
\hline 13.0 & 365 & 28 \\
\hline 13.0 & 600 & 46 \\
\hline 13.0 & 400 & 31 \\
\hline 15.1 & 600 & 40 \\
\hline 15.1 & 650 & 43 \\
\hline 15.1 & 900 & 60 \\
\hline 15.1 & 630 & 42 \\
\hline 15.1 & 745 & 49 \\
\hline 7.5 & 150 & 20 \\
\hline 7.5 & 250 & 33 \\
\hline 7.5 & 150 & 20 \\
\hline 7.5 & 230 & 31 \\
\hline 7.5 & 330 & 44 \\
\hline 13.0 & 425 & 33 \\
\hline 13.0 & 400 & 31 \\
\hline 13.0 & 700 & 54 \\
\hline 13.0 & 680 & 52 \\
\hline 13.0 & 825 & 63 \\
\hline 15.1 & 888 & 59 \\
\hline 15.1 & 925 & 61 \\
\hline
\end{tabular}




\begin{tabular}{|c|c|c|}
\hline 15.1 & 1000 & 66 \\
\hline 15.1 & 760 & 50 \\
\hline 15.1 & 800 & 53 \\
\hline 6.9 & 549 & 79 \\
\hline 6.9 & 429 & 62 \\
\hline 6.9 & 535 & 77 \\
\hline 6.9 & 503 & 73 \\
\hline 6.9 & 505 & 73 \\
\hline 6.9 & 502 & 73 \\
\hline 13.1 & 666 & 51 \\
\hline 13.1 & 874 & 67 \\
\hline 13.1 & 819 & 62 \\
\hline 13.1 & 820 & 62 \\
\hline 13.1 & 681 & 52 \\
\hline 13.1 & 775 & 59 \\
\hline 13.1 & 642 & 49 \\
\hline 15.5 & 663 & 43 \\
\hline 15.5 & 653 & 42 \\
\hline 15.5 & 1005 & 65 \\
\hline 15.5 & 1063 & 69 \\
\hline 15.5 & 1031 & 67 \\
\hline 15.5 & 1031 & 67 \\
\hline 15.5 & 790 & 51 \\
\hline 6.9 & 425 & 61 \\
\hline 6.9 & 509 & 74 \\
\hline 6.9 & 316 & 46 \\
\hline 6.9 & 437 & 63 \\
\hline 6.9 & 600 & 87 \\
\hline 6.9 & 513 & 74 \\
\hline 6.9 & 426 & 62 \\
\hline 6.9 & 482 & 70 \\
\hline 6.9 & 523 & 76 \\
\hline 6.9 & 538 & 78 \\
\hline 6.9 & 500 & 72 \\
\hline 6.9 & 420 & 61 \\
\hline 13.1 & 511 & 39 \\
\hline 13.1 & 439 & 33 \\
\hline 13.1 & 266 & 20 \\
\hline 13.1 & 665 & 51 \\
\hline 13.1 & 646 & 49 \\
\hline 13.1 & 578 & 44 \\
\hline
\end{tabular}




\begin{tabular}{|c|c|c|}
\hline 13.1 & 494 & 38 \\
\hline 13.1 & 448 & 34 \\
\hline 13.1 & 434 & 33 \\
\hline 13.1 & 438 & 33 \\
\hline 13.1 & 483 & 37 \\
\hline 13.1 & 688 & 52 \\
\hline 15.5 & 711 & 46 \\
\hline 15.5 & 1245 & 80 \\
\hline 15.5 & 1207 & 78 \\
\hline 15.5 & 1160 & 75 \\
\hline 15.5 & 1030 & 66 \\
\hline 15.5 & 735 & 47 \\
\hline 15.5 & 784 & 51 \\
\hline 15.5 & 1024 & 66 \\
\hline 15.5 & 870 & 56 \\
\hline 15.5 & 656 & 42 \\
\hline 15.5 & 870 & 56 \\
\hline 15.5 & 1001 & 65 \\
\hline 3.2 & 663 & 208 \\
\hline 3.2 & 594 & 186 \\
\hline 3.2 & 523 & 164 \\
\hline 3.2 & 595 & 187 \\
\hline 3.2 & 440 & 138 \\
\hline 3.2 & 435 & 136 \\
\hline 5.6 & 752 & 135 \\
\hline 5.6 & 833 & 149 \\
\hline 5.6 & 767 & 137 \\
\hline 5.6 & 900 & 161 \\
\hline 5.6 & 519 & 93 \\
\hline 5.6 & 588 & 105 \\
\hline 5.6 & 429 & 77 \\
\hline 6.5 & 755 & 117 \\
\hline 6.5 & 663 & 103 \\
\hline 6.5 & 916 & 142 \\
\hline 6.5 & 1024 & 159 \\
\hline 6.5 & 626 & 97 \\
\hline 6.5 & 911 & 141 \\
\hline 3.2 & 586 & 184 \\
\hline 3.2 & 539 & 169 \\
\hline 3.2 & 510 & 160 \\
\hline 3.2 & 461 & 145 \\
\hline
\end{tabular}




\begin{tabular}{|c|c|c|}
\hline 3.2 & 418 & 131 \\
\hline 3.2 & 404 & 127 \\
\hline 5.6 & 832 & 149 \\
\hline 5.6 & 898 & 161 \\
\hline 5.6 & 610 & 109 \\
\hline 5.6 & 591 & 106 \\
\hline 5.6 & 891 & 159 \\
\hline 5.6 & 745 & 133 \\
\hline 6.5 & 693 & 107 \\
\hline 6.5 & 1143 & 177 \\
\hline 6.5 & 859 & 133 \\
\hline 6.5 & 811 & 126 \\
\hline 6.5 & 931 & 144 \\
\hline 6.5 & 900 & 139 \\
\hline 6.1 & 707 & 116 \\
\hline 6.1 & 675 & 111 \\
\hline 6.1 & 586 & 96 \\
\hline 6.1 & 750 & 123 \\
\hline 6.1 & 625 & 103 \\
\hline 6.1 & 592 & 97 \\
\hline 10.8 & 883 & 82 \\
\hline 10.8 & 704 & 65 \\
\hline 10.8 & 704 & 65 \\
\hline 10.8 & 834 & 77 \\
\hline 10.8 & 974 & 91 \\
\hline 10.8 & 799 & 74 \\
\hline 13.2 & 1005 & 76 \\
\hline 13.2 & 979 & 74 \\
\hline 13.2 & 1001 & 76 \\
\hline 13.2 & 807 & 61 \\
\hline 13.2 & 1208 & 91 \\
\hline 13.2 & 888 & 67 \\
\hline 6.1 & 307 & 51 \\
\hline 6.1 & 535 & 88 \\
\hline 6.1 & 439 & 72 \\
\hline 6.1 & 601 & 99 \\
\hline 6.1 & 478 & 79 \\
\hline 6.1 & 596 & 98 \\
\hline 10.8 & 821 & 76 \\
\hline 10.8 & 1008 & 94 \\
\hline 10.8 & 989 & 92 \\
\hline
\end{tabular}




\begin{tabular}{|c|c|c|}
\hline 10.8 & 802 & 75 \\
\hline 10.8 & 805 & 75 \\
\hline 10.8 & 819 & 76 \\
\hline 13.2 & 1009 & 76 \\
\hline 13.2 & 890 & 67 \\
\hline 13.2 & 1189 & 90 \\
\hline 13.2 & 1251 & 94 \\
\hline 13.2 & 961 & 73 \\
\hline 13.2 & 1131 & 85 \\
\hline 3.5 & 591 & 167 \\
\hline 3.5 & 617 & 174 \\
\hline 3.5 & 438 & 124 \\
\hline 3.5 & 667 & 188 \\
\hline 3.5 & 662 & 187 \\
\hline 3.5 & 454 & 128 \\
\hline 5.9 & 1000 & 168 \\
\hline 5.9 & 766 & 129 \\
\hline 5.9 & 1001 & 168 \\
\hline 5.9 & 989 & 166 \\
\hline 5.9 & 762 & 128 \\
\hline 5.9 & 999 & 168 \\
\hline 7.0 & 850 & 122 \\
\hline 7.0 & 968 & 139 \\
\hline 7.0 & 819 & 118 \\
\hline 7.0 & 876 & 126 \\
\hline 7.0 & 743 & 107 \\
\hline 7.0 & 681 & 98 \\
\hline 3.5 & 529 & 149 \\
\hline 3.5 & 506 & 143 \\
\hline 3.5 & 358 & 101 \\
\hline 3.5 & 623 & 176 \\
\hline 3.5 & 580 & 164 \\
\hline 3.5 & 428 & 121 \\
\hline 5.9 & 827 & 139 \\
\hline 5.9 & 550 & 92 \\
\hline 5.9 & 926 & 156 \\
\hline 5.9 & 840 & 141 \\
\hline 5.9 & 598 & 101 \\
\hline 5.9 & 999 & 168 \\
\hline 7.0 & 833 & 120 \\
\hline 7.0 & 674 & 97 \\
\hline
\end{tabular}




\begin{tabular}{|c|c|c|}
\hline 7.0 & 1017 & 146 \\
\hline 7.0 & 991 & 142 \\
\hline 7.0 & 614 & 88 \\
\hline 7.0 & 588 & 85 \\
\hline 7.0 & 675 & 97 \\
\hline 9.3 & 1083 & 116 \\
\hline 9.3 & 1142 & 123 \\
\hline 9.3 & 685 & 73 \\
\hline 9.3 & 692 & 74 \\
\hline 9.3 & 1122 & 120 \\
\hline 9.3 & 915 & 98 \\
\hline 6.8 & 825 & 122 \\
\hline 6.8 & 580 & 86 \\
\hline 6.8 & 905 & 133 \\
\hline 6.8 & 720 & 106 \\
\hline 6.8 & 420 & 62 \\
\hline 6.8 & 900 & 133 \\
\hline 6.8 & 845 & 125 \\
\hline 6.8 & 430 & 63 \\
\hline 6.8 & 575 & 85 \\
\hline 6.8 & 570 & 84 \\
\hline & & \\
\hline
\end{tabular}




\section{$1000 \mu \mathrm{g} \quad 3000 \mu \mathrm{gg} \quad 5000 \mu \mathrm{g}$}

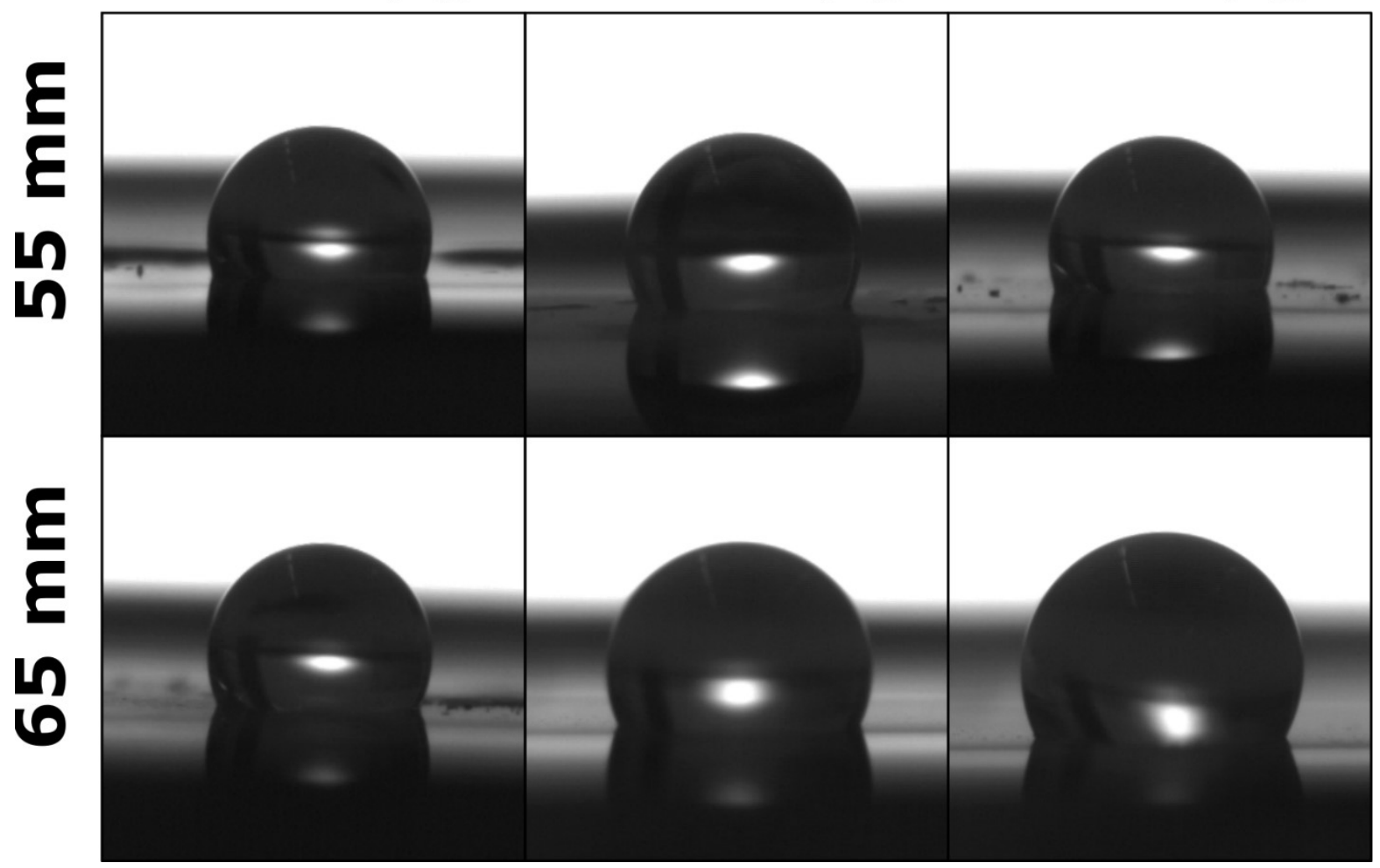

Figure S1: Representative images of sessile droplets of water on electrospray-printed polyimide films. Films were printed at separation distances of 55 and $65 \mathrm{~mm}$ with sprayed masses of 1000, 3000, and $5000 \mu \mathrm{g}$.

Table S3: Contact angle (wettability) testing raw data.

\begin{tabular}{|c|c|c|c|}
\hline Sprayed Mass $[\boldsymbol{\mu g}]$ & Film Thickness $[\boldsymbol{\mu m}]$ & Separation Distance $[\mathbf{m m}]$ & Contact Angle $\left[^{\circ}{ }^{\mathbf{m}}\right.$ \\
\hline 1000 & 4.1 & 65 & 118 \\
\hline 1000 & 4.1 & 65 & 120 \\
\hline 1000 & 4.1 & 65 & 118 \\
\hline 1000 & 4.1 & 65 & 121 \\
\hline 1000 & 4.1 & 65 & 120 \\
\hline 3000 & 6.0 & 65 & 118 \\
\hline 3000 & 6.0 & 65 & 119 \\
\hline 3000 & 6.0 & 65 & 121 \\
\hline 3000 & 6.0 & 65 & 120 \\
\hline 3000 & 6.0 & 65 & 121 \\
\hline 5000 & 7.1 & 65 & 117 \\
\hline 5000 & 7.1 & 65 & 120 \\
\hline 5000 & 7.1 & 65 & 120 \\
\hline
\end{tabular}




\begin{tabular}{|c|c|c|c|}
\hline 5000 & 7.1 & 65 & 117 \\
\hline 5000 & 7.1 & 65 & 117 \\
\hline 1000 & 4.1 & 65 & 104 \\
\hline 1000 & 4.1 & 65 & 109 \\
\hline 1000 & 4.1 & 65 & 110 \\
\hline 1000 & 4.1 & 65 & 105 \\
\hline 1000 & 4.1 & 65 & 111 \\
\hline 3000 & 6.0 & 65 & 111 \\
\hline 3000 & 6.0 & 65 & 113 \\
\hline 3000 & 6.0 & 65 & 111 \\
\hline 3000 & 6.0 & 65 & 114 \\
\hline 3000 & 6.0 & 65 & 115 \\
\hline 5000 & 7.1 & 65 & 111 \\
\hline 5000 & 7.1 & 65 & 110 \\
\hline 5000 & 7.1 & 65 & 115 \\
\hline 5000 & 7.1 & 65 & 113 \\
\hline 5000 & 7.1 & 65 & 111 \\
\hline 1000 & 7.5 & 55 & 127 \\
\hline 1000 & 7.5 & 55 & 124 \\
\hline 1000 & 7.5 & 55 & 117 \\
\hline 1000 & 7.5 & 55 & 121 \\
\hline 1000 & 7.5 & 55 & 102 \\
\hline 3000 & 13.0 & 55 & 119 \\
\hline 3000 & 13.0 & 55 & 121 \\
\hline 3000 & 13.0 & 55 & 100 \\
\hline 3000 & 13.0 & 55 & 121 \\
\hline 3000 & 13.0 & 55 & 124 \\
\hline 5000 & 15.1 & 55 & 100 \\
\hline 5000 & 15.1 & 55 & 119 \\
\hline 5000 & 15.1 & 55 & 121 \\
\hline 5000 & 15.1 & 55 & 116 \\
\hline 5000 & 15.1 & 55 & 127 \\
\hline 1000 & 7.5 & 55 & 94 \\
\hline 1000 & 7.5 & 55 & 100 \\
\hline 1000 & 7.5 & 55 & 103 \\
\hline 1000 & 7.5 & 55 & 106 \\
\hline 1000 & 7.5 & 55 & 103 \\
\hline 3000 & 13.0 & 55 & 108 \\
\hline 3000 & 13.0 & 55 & 109 \\
\hline 3000 & 13.0 & 55 & 102 \\
\hline 3000 & 13.0 & 55 & 114 \\
\hline
\end{tabular}




\begin{tabular}{|c|c|c|c|}
\hline 3000 & 13.0 & 55 & 105 \\
\hline 5000 & 15.1 & 55 & 99 \\
\hline 5000 & 15.1 & 55 & 105 \\
\hline 5000 & 15.1 & 55 & 101 \\
\hline 5000 & 15.1 & 55 & 107 \\
\hline 5000 & 15.1 & 55 & 98 \\
\hline 1000 & 6.9 & 55 & 95 \\
\hline 1000 & 6.9 & 55 & 117 \\
\hline 1000 & 6.9 & 55 & 118 \\
\hline 1000 & 6.9 & 55 & 91 \\
\hline 1000 & 6.9 & 55 & 115 \\
\hline 1000 & 6.9 & 55 & 119 \\
\hline 3000 & 13.1 & 55 & 112 \\
\hline 3000 & 13.1 & 55 & 118 \\
\hline 3000 & 13.1 & 55 & 122 \\
\hline 3000 & 13.1 & 55 & 90 \\
\hline 3000 & 13.1 & 55 & 115 \\
\hline 3000 & 13.1 & 55 & 120 \\
\hline 5000 & 15.5 & 55 & 103 \\
\hline 5000 & 15.5 & 55 & 111 \\
\hline 5000 & 15.5 & 55 & 107 \\
\hline 5000 & 15.5 & 55 & 93 \\
\hline 5000 & 15.5 & 55 & 105 \\
\hline 5000 & 15.5 & 55 & 108 \\
\hline 1000 & 6.9 & 55 & 90 \\
\hline 1000 & 6.9 & 55 & 120 \\
\hline 1000 & 6.9 & 55 & 106 \\
\hline 1000 & 6.9 & 55 & 121 \\
\hline 1000 & 6.9 & 55 & 116 \\
\hline 1000 & 6.9 & 55 & 113 \\
\hline 3000 & 13.1 & 55 & 120 \\
\hline 3000 & 13.1 & 55 & 113 \\
\hline 3000 & 13.1 & 55 & 109 \\
\hline 3000 & 13.1 & 55 & 108 \\
\hline 3000 & 13.1 & 55 & 112 \\
\hline 3000 & 13.1 & 55 & 116 \\
\hline 5000 & 15.5 & 55 & 116 \\
\hline 5000 & 15.5 & 55 & 124 \\
\hline 5000 & 15.5 & 55 & 105 \\
\hline 5000 & 15.5 & 55 & 120 \\
\hline 5000 & 15.5 & 55 & 105 \\
\hline
\end{tabular}




\begin{tabular}{|c|c|c|c|}
\hline 5000 & 15.5 & 55 & 118 \\
\hline 1000 & 3.2 & 65 & 115 \\
\hline 1000 & 3.2 & 65 & 111 \\
\hline 1000 & 3.2 & 65 & 90 \\
\hline 1000 & 3.2 & 65 & 97 \\
\hline 1000 & 3.2 & 65 & 122 \\
\hline 1000 & 3.2 & 65 & 105 \\
\hline 3000 & 5.6 & 65 & 97 \\
\hline 3000 & 5.6 & 65 & 94 \\
\hline 3000 & 5.6 & 65 & 115 \\
\hline 3000 & 5.6 & 65 & 111 \\
\hline 3000 & 5.6 & 65 & 97 \\
\hline 3000 & 5.6 & 65 & 85 \\
\hline 5000 & 6.5 & 65 & 115 \\
\hline 5000 & 6.5 & 65 & 104 \\
\hline 5000 & 6.5 & 65 & 100 \\
\hline 5000 & 6.5 & 65 & 114 \\
\hline 5000 & 6.5 & 65 & 121 \\
\hline 5000 & 6.5 & 65 & 108 \\
\hline 1000 & 3.2 & 65 & 123 \\
\hline 1000 & 3.2 & 65 & 125 \\
\hline 1000 & 3.2 & 65 & 125 \\
\hline 1000 & 3.2 & 65 & 124 \\
\hline 1000 & 3.2 & 65 & 122 \\
\hline 1000 & 3.2 & 65 & 115 \\
\hline 3000 & 5.6 & 65 & 116 \\
\hline 3000 & 5.6 & 65 & 122 \\
\hline 3000 & 5.6 & 65 & 121 \\
\hline 3000 & 5.6 & 65 & 123 \\
\hline 3000 & 5.6 & 65 & 120 \\
\hline 3000 & 5.6 & 65 & 123 \\
\hline 5000 & 6.5 & 65 & 116 \\
\hline 5000 & 6.5 & 65 & 119 \\
\hline 5000 & 6.5 & 65 & 124 \\
\hline 5000 & 6.5 & 65 & 124 \\
\hline 5000 & 6.5 & 65 & 121 \\
\hline 5000 & 6.5 & 65 & 117 \\
\hline 1000 & 6.1 & 55 & 127 \\
\hline 1000 & 6.1 & 55 & 123 \\
\hline 1000 & 6.1 & 55 & 115 \\
\hline 1000 & 6.1 & 55 & 124 \\
\hline
\end{tabular}




\begin{tabular}{|c|c|c|c|}
\hline 1000 & 6.1 & 55 & 123 \\
\hline 1000 & 6.1 & 55 & 122 \\
\hline 3000 & 10.8 & 55 & 120 \\
\hline 3000 & 10.8 & 55 & 118 \\
\hline 3000 & 10.8 & 55 & 122 \\
\hline 3000 & 10.8 & 55 & 128 \\
\hline 3000 & 10.8 & 55 & 113 \\
\hline 3000 & 10.8 & 55 & 125 \\
\hline 5000 & 13.2 & 55 & 115 \\
\hline 5000 & 13.2 & 55 & 121 \\
\hline 5000 & 13.2 & 55 & 116 \\
\hline 5000 & 13.2 & 55 & 122 \\
\hline 5000 & 13.2 & 55 & 123 \\
\hline 5000 & 13.2 & 55 & 123 \\
\hline 1000 & 6.1 & 55 & 117 \\
\hline 1000 & 6.1 & 55 & 100 \\
\hline 1000 & 6.1 & 55 & 88 \\
\hline 1000 & 6.1 & 55 & 124 \\
\hline 1000 & 6.1 & 55 & 119 \\
\hline 1000 & 6.1 & 55 & 108 \\
\hline 3000 & 10.8 & 55 & 119 \\
\hline 3000 & 10.8 & 55 & 109 \\
\hline 3000 & 10.8 & 55 & 110 \\
\hline 3000 & 10.8 & 55 & 115 \\
\hline 3000 & 10.8 & 55 & 102 \\
\hline 3000 & 10.8 & 55 & 109 \\
\hline 5000 & 13.2 & 55 & 122 \\
\hline 5000 & 13.2 & 55 & 117 \\
\hline 5000 & 13.2 & 55 & 106 \\
\hline 5000 & 13.2 & 55 & 109 \\
\hline 5000 & 13.2 & 55 & 98 \\
\hline 5000 & 13.2 & 55 & 100 \\
\hline 1000 & 3.5 & 65 & 109 \\
\hline 1000 & 3.5 & 65 & 110 \\
\hline 1000 & 3.5 & 65 & 119 \\
\hline 1000 & 3.5 & 65 & 122 \\
\hline 1000 & 3.5 & 65 & 118 \\
\hline 1000 & 3.5 & 65 & 122 \\
\hline 3000 & 5.9 & 65 & 119 \\
\hline 3000 & 5.9 & 65 & 94 \\
\hline 3000 & 5.9 & 65 & 109 \\
\hline
\end{tabular}




\begin{tabular}{|c|c|c|c|}
\hline 3000 & 5.9 & 65 & 104 \\
\hline 3000 & 5.9 & 65 & 108 \\
\hline 3000 & 5.9 & 65 & 114 \\
\hline 5000 & 7.0 & 65 & 118 \\
\hline 5000 & 7.0 & 65 & 110 \\
\hline 5000 & 7.0 & 65 & 111 \\
\hline 5000 & 7.0 & 65 & 90 \\
\hline 5000 & 7.0 & 65 & 119 \\
\hline 5000 & 7.0 & 65 & 115 \\
\hline 1000 & 3.5 & 65 & 108 \\
\hline 1000 & 3.5 & 65 & 101 \\
\hline 1000 & 3.5 & 65 & 96 \\
\hline 1000 & 3.5 & 65 & 90 \\
\hline 1000 & 3.5 & 65 & 107 \\
\hline 1000 & 3.5 & 65 & 96 \\
\hline 3000 & 5.9 & 65 & 112 \\
\hline 3000 & 5.9 & 65 & 101 \\
\hline 3000 & 5.9 & 65 & 106 \\
\hline 3000 & 5.9 & 65 & 109 \\
\hline 3000 & 5.9 & 65 & 113 \\
\hline 3000 & 5.9 & 65 & 112 \\
\hline 5000 & 7.0 & 65 & 100 \\
\hline 5000 & 7.0 & 65 & 106 \\
\hline 5000 & 7.0 & 65 & 103 \\
\hline 5000 & 7.0 & 65 & 110 \\
\hline 5000 & 7.0 & 65 & 106 \\
\hline 5000 & 7.0 & 65 & 110 \\
\hline 5000 & 6.8 & 65 & 119 \\
\hline 5000 & 6.8 & 65 & 119 \\
\hline 5000 & 6.8 & 65 & 119 \\
\hline 5000 & 6.8 & 65 & 117 \\
\hline 5000 & 6.8 & 65 & 121 \\
\hline 5000 & 6.8 & 65 & 119 \\
\hline 5000 & 6.8 & 65 & 108 \\
\hline 5000 & 6.8 & 65 & 109 \\
\hline 5000 & 6.8 & 65 & 111 \\
\hline 5000 & 6.8 & 65 & 112 \\
\hline 5000 & 6.8 & 65 & 109 \\
\hline 5000 & 6.8 & 65 & 111 \\
\hline
\end{tabular}

